\title{
Modélisation régionale des régimes de crue du bassin hydrographique de la Moselle française
}

\author{
Floods regional modelling in the catchment area \\ of the French Moselle
}

G. Galéa*, M. Blum

Cemagref-Lyon, Unité de Recherche Hydrologie Hydraulique, 3 bis quai Chauveau 69336 Lyon Cedex 09

The studied area is the Moselle basin. Available data were collected within the framework of an European project (NOAH) and a French national project (PACTES). Among these 59 available sub-catchments, only 53 of them were selected to calibrate and validate local and regional statistical flood models. The corresponding models are elaborated from a statistical model flow-duration-frequency $(Q d F)$ with 3 parameters using the observed affinity property of distributions. An extrapolation of the QdF model to the flood quantiles of high return periods is possible using the GRADEX methodology. The duration $\Delta$ represents a characteristic flood duration of the basin and thus informs about the more or less high celerity of the flow. This duration $\Delta$ as well as the 10-year peak discharge Q10 represent the two local descriptors of the regime to be known. They are the inputs of the regional QdF model, which allows the flood regime to be estimated at any target site, at which few or no observation exists. When Q10 and $\Delta$ are deduced from the flow observation, the comparison with local modeling showed the robustness of the established regional model. This indicates that the studied geographical area is relatively quite homogeneous, at least concerning the validity of the regional QdF model. On the other hand, when $Q 10$ and $\Delta$ are estimated by classical multiple regressions (calibration set, validation set), estimates can contain strong errors that significantly affect the results of the regional model. To improve the results of the linear model, an approach by canonical correlation analysis was carried out. This technique leads to the identification of a neighbourhood in a space of canonical variables. Comparatively to the traditional method, this approach clearly improves the estimate of local descriptors. Mean quadratic error is improved by $50 \%$ for Q10 and by $33 \%$ for $\Delta$. For an easy use, the regional QdF model is presented under its two formalisms: First one for small return periods and second one for higher ones.

Key Words: flood, statistical hydrology, regionalization, canonical correlation analysis.

\section{I $\square$ CONTEXTE HISTORIQUE}

Notre démarche s'inscrit dans la continuité des travaux menés depuis quelques années dans la zone Moselle. Nous citerons le projet européen NOAH [2000] et le projet national PACTES [2001]. Dans le cadre de NOAH s'inscrivent comme principaux travaux ceux de Charleux-Demargne [2001] et de Javelle [2001]. Pour ce qui concerne PACTES, la tendance a été de valoriser sur le plan opérationnel les acquis de ces travaux de thèses. Par ailleurs, des travaux d'homogénéisation de l'information collectée et élaborée dans NOAH et PACTES ont été réalisés [Galéa et al., 2001] et poursuivis dans le cadre du développement de l'outil SIG par Vanderbecq [2003] et de la recherche menée par Blum [2003].

\section{II $\square$ BUT DE NOTRE PROPOS}

Présenter le modèle statistique régional des régimes de crue et son incertitude sur l'estimation des quantiles de crue [Galéa et al., 2001]. Montrer la réduction significative de cette incertitude grâce à l'identification, par l'analyse de corrélation canonique, des voisinages hydrologiques homogènes des descripteurs de régime locaux $Q 10$ et $\Delta$ 
[Blum, 2003]. Ces descripteurs, comme nous le verrons ultérieurement, sont les entrées du modèle statistique régional, respectivement le débit de pointe décennal et un temps de transfert.

\section{III 口PRÉSENTATION DU BASSIN HYDROGRAPHIQUE DE LA MOSELLE FRANÇAISE ET DES SOUS-BASSINS INVENTORIÉS}

Le bassin hydrographique de la Moselle couvre une superficie totale d'environ $28152 \mathrm{~km}^{2}$, dont $16454 \mathrm{~km}^{2}$ en France. Cette zone relativement restreinte présente une assez grande diversité des écoulements représentée par 59 sousbassins de superficie très variable, de $16 \mathrm{~km}^{2}$ à $9395 \mathrm{~km}^{2}$ (fig. 1). Le contexte historique des études menées sur le bassin hydrographique de la Moselle nous a permis de disposer sur ces 59 sites des chroniques de débit $Q(t)$ ainsi que des caractéristiques statistiques des pluies maximales de bassin de différentes durées. Ces pluies quotidiennes de bassin ont été estimées à partir de 187 postes pluviométriques suivis par METEO France [Javelle, 2001]. Parmi ces 59 sous-bassins disponibles, seuls 53 d'entre eux ont été retenus pour caler et valider le modèle régional. Nous avons considéré un jeu de calage de 27 sous-bassins et de 12 sous-bassins pour la validation. Le choix de ces deux jeux de sous-bassins a privilégié à la fois des écoulements non influencés et indépendants. Par souci opérationnel nous avons aussi considéré un jeu de validation complémentaire de 14 sous-bassins, d'écoulements non indépendants et plus ou moins influencés.

Les classes de superficie réparties dans les trois jeux de sous-bassins sont présentées à la figure 2 .



Figure 1 : Le bassin hydrographique de la Moselle et ses 59 sous-bassins inventoriés.
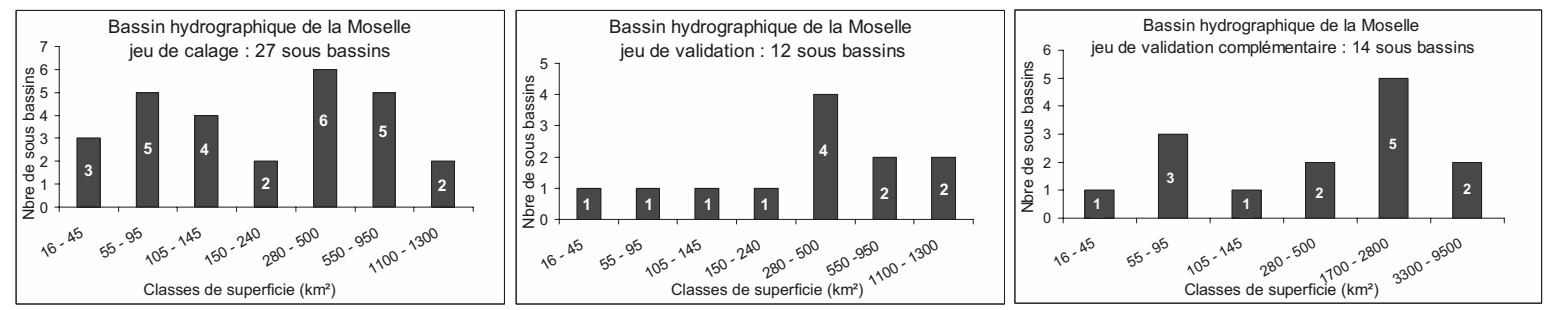

Figure 2 : Classes de superficie des sous-bassins retenus.

\section{ANALYSE DES CRUES DE FAIBLE PÉRIODE DE RETOUR 0,5 $\leq T(A N) \leq 20$}

\section{IV.1 Modélisation QdF locale}

L'analyse débit-durée-fréquence des chroniques de débit observées $Q(t)$ permet une description théorique multi-durée des quantiles de crue. Cette dernière est formalisée selon un modèle QdF dont le calage local exploite l'affinité observée des distributions de différentes durées $d$ étudiées [Javelle, 2001].

\section{IV.1.1 Définition des variables hydrologiques}

La variabilité temporelle des crues $Q(t)$ est étudiée à partir de deux variables hydrologiques notées $V d$ et $Q d: V d$ est le débit moyen sur la durée continue $d$, maximal lors de l'épisode de crue, et $Q d$ représente le débit seuil, continûment dépassé sur la durée $d$, maximal lors de l'épisode de crue. Lorsque $d=0$, les deux variables sont égales au débit de pointe maximal $Q I X$ de l'épisode (fig. 3).

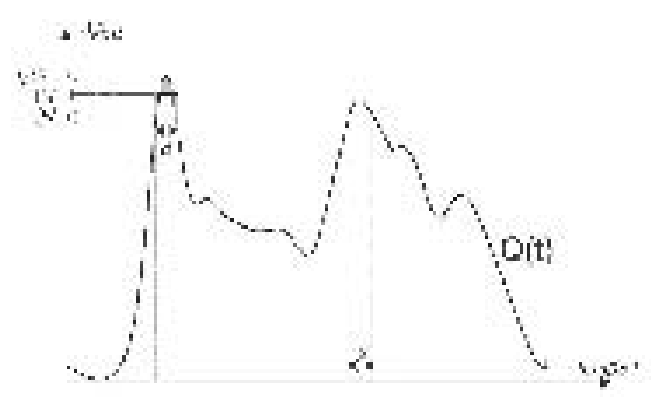

Figure 3 : Définition des variables hydrologiques d'un épisode de crue. 


\section{IV.1.2 Ajustement statistique: loi exponentielle}

La loi de probabilité choisie (1), pour chaque échantillon de durée $d$ constitué, est une loi exponentielle ajustée a un échantillon de valeurs $V d$ (ou $Q d$ ) supérieures à un seuil dont le processus d'occurrence suit une loi de Poisson [Lang et al., 1999].

$$
V_{d}(T)=G q_{d} \cdot \ln (T)+Q 0_{d}
$$

où $G q_{d}$ est le gradex des débits (ou encore paramètre d'échelle de la loi), $Q 0_{d}$ est le paramètre de position de la loi et $T(\mathrm{an})=n a / n s\left(1-F_{i}\right)$ avec na le nombre d'années d'observation et $n s$ le nombre de valeurs sélectionnées. $F_{i}$ est la fréquence au non dépassement où $i$ est le rang $\left(F_{i}=\frac{i-0,3}{n s+0,4}\right), G q_{d}=m-V_{i=1}$, où $m$ et $V_{i=1}$ sont respectivement la moyenne des débits et le plus petit des débits, et $Q 0_{d}=V_{i=1}+G q_{d} \cdot \ln (n s / n a)$.

\section{IV.1.3 Modèle QdF convergent local}

La propriété de convergence observée des distributions (1) vers les faibles périodes de retour permet d'élaborer, d'après Javelle et al. [1999, 2000], un modèle QdF dit « convergent». Le concept de modélisation utilise la propriété d'affinité des distributions et permet de proposer un modèle $\mathrm{QdF}$ à faible nombre de paramètres. Les 2 paramètres de la loi exponentielle (2) ainsi que $\Delta$ sont ajustés sur l'ensemble des quantiles expérimentaux relatifs aux durées $d$ étudiées. Par ailleurs, lorsque $d$ tend vers l'infini, les distributions de durée $d$ tendent vers une distribution limite théorique d'ordonnée $\overline{Q A}\left(\lim _{d \rightarrow \infty}\left(V_{L}(d, T)\right)=\overline{Q A}\right)$. Nous noterons $V_{L}(d, T)$ les quantiles déduits du modèle $\mathrm{QdF}$ local à 4 paramètres (2), avec $V_{L}(0, T)=a(0) \cdot \ln (T)+X 0(0)$ la distribution consolidée des débits de pointe (QIX), $\overline{Q A}$ : le module inter-annuel et $\Delta$ une durée caractéristique de crue du bassin versant.

$$
V_{L}(d, T)=\frac{V_{L}(d=0, T)-\overline{Q A}}{1+d / \Delta}+\overline{Q A}
$$

L'expression du modèle QdF à 4 paramètres, pour le sousbassin du Madon à Mirecourt (fig. 1, $\mathrm{n}^{\circ} 12$ ), sera par exemple donnée par (3). Les distributions théoriques ainsi que les quantiles expérimentaux sont présentés à la figure 4.

$$
V_{L}(d, T)=\frac{31,1 \ln (T)+77,8-4,7}{1+d / 2,33}+4,7
$$

Lors de la régionalisation des crues du bassin hydrographique de la Moselle [Galéa et al., 2001] l'expression (2) du modèle QdF a pu être simplifiée et son nombre de paramètres réduit à trois : $a(0), X 0(0)$ et $\Delta$. Par ailleurs, cette modélisation locale (2) qui permet une consolidation des ajustements (1) avec la durée $d$ sera assimilée ultérieurement à la référence «observation» lors du test d'erreur quadratique effectué sur les quantiles régionaux.

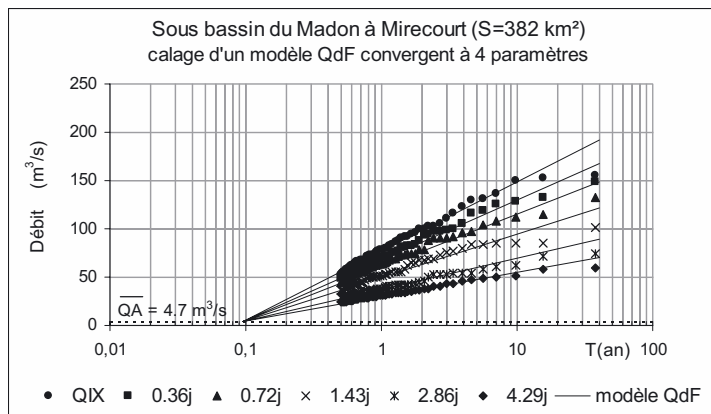

Figure 4 : Exemple de représentation selon (3).

\section{IV.2 Modélisation QdF régionale}

Le modèle $\mathrm{QdF}$ régional est établi à partir des paramètres $a(0)$ et $X 0(0)$ calés en chacun des 27 sous-bassins du jeu de calage. Ces paramètres sont normés par le débit de pointe décennal $Q 10$, et leurs moyennes respectives [Dalrymple, 1960] permettent de définir les paramètres régionaux $a_{R}(0)$ et $X 0_{R}(0)$. Le modèle $\mathrm{QdF}$ régional (4) permet de caractériser le régime des crues d'un site cible dont les descripteurs de régime sont connus, déduits d'observations de débit ou estimés. Les quantiles de crue seront notés $V_{R}(d, T)$.

$$
V_{R}(d, T)=\left[\frac{0,209 \ln T+0,526}{1+d / \Delta_{\text {site cible }}}\right] \cdot Q 10_{\text {site cible }}
$$

L'équation (4) permet le calcul des quantiles de crue de durée $d$ compatible avec la dynamique de crue du sous-bassin $(0 \leq d(\mathrm{j}) \leq 2 \Delta)$ et pour des périodes moyennes de retour $\mathrm{T}$ comprises entre deux fois par an et 20 ans $(0,5 \leq T(\operatorname{an}) \leq 20)$. Vingt ans est une limite supérieure acceptable de la validité de (4) qui est de 10 ans, seuil fréquentiel d'extrapolation aux grandes périodes de retour.

\section{ANALYSE DES CRUES DE FRÉQUENCE RARE $(10<T(\mathrm{AN}) \leq 1000)$}

\section{V.1 Modélisation QdF locale}

La distribution théorique relative aux observations (1) peut être extrapolée aux quantiles de débit de grande période de retour selon une variante du GRADEX [Guillot et Duband, 1967], proposée par Michel et Oberlin [1987]. La forme d'extrapolation (5) concerne des durées $d$ compatibles avec la dynamique de crue des sous-bassins $(\Delta / 3 \leq d \leq 1,5 \Delta)$.

$$
V_{d}(T)=V_{d}(10)+G p_{d} \cdot \ln \left[1+\frac{G q_{d}}{G p_{d}} \cdot \frac{T-10}{10}\right]
$$

où $V_{d}(10)$ est déduit de (1) et $G p_{d}$ représente le gradex des pluies maximales de bassin observées [Javelle, 2001] ajustées selon une expression équivalente à (1). $G p_{d}$ est exprimé en $\mathrm{m}^{3} / \mathrm{s}$ en faisant intervenir la superficie du sous-bassin. Le passage aux quantiles de pointe (6) privilégie la distribution de débit relative à $d=\Delta / 3$ et un coefficient de forme moyen $\bar{r}$ (avec $r=Q I X / V_{d}$ ). 


$$
Q I X(T)=V_{d}(T) \cdot \bar{r}
$$

Les quantiles de crue obtenus selon (5) et (6) vont permettre une extrapolation du modèle QdF (2) aux grandes périodes de retour (7).

$$
V_{L}(d, T)=V_{L}(d, 10)+c(d) \cdot \ln \left[1+\frac{a(d)}{c(d)} \cdot \frac{T-10}{10}\right]
$$

avec $a(d)=\frac{a(0)}{1+\frac{d}{\Delta}}$, gradex des débits de durée $d$ modélisé (2), et $c(d)=\frac{1}{p_{1} \cdot d+p_{2}}$, fonction de généralisation de $G p_{d}$ avec $d$, où $p_{1}$ et $p_{2}$ sont des paramètres calés sur l'ensemble des quantiles de crue ( $T \geq 20$ ans).

\section{V.2 Modélisation QdF régionale}

L'application de la norme $Q 10$ à l'expression (7) permet de caler une fonction implicite du gradex des pluies (8) pour chaque sous-bassin du jeu de calage.

$$
c^{*}(d)=\frac{1}{p_{1}^{*} \cdot \frac{d}{\Delta}+p_{2}^{*}}
$$

Si l'on représente sur un même graphe (fig. 5) la variation du gradex implicite $c^{*}(d)$ des pluies des 27 sous-bassins, nous pouvons en déduire par optimisation une fonction du gradex régional des pluies maximales (9).

$$
c_{R}(d)=\frac{1}{4,29 \frac{d}{\Delta}+2,71}
$$

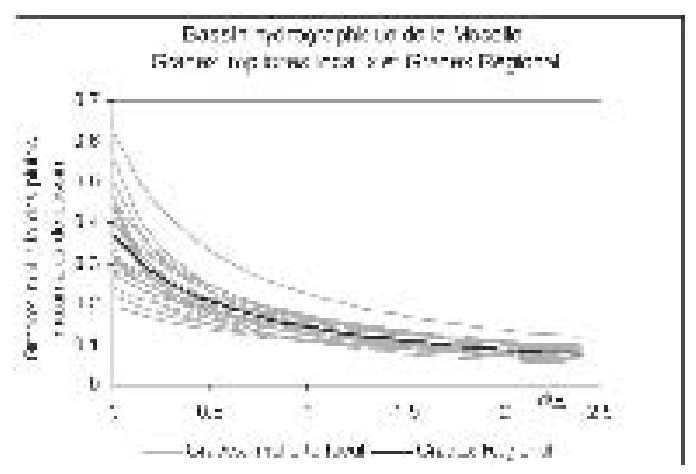

Figure 5 : Gradex des pluies implicite local et régional.

Les quantiles de crue de fréquence rare, en un site cible, pourront être déduits de l'expression (10).

$$
V_{R}(d, T)=V_{R}(d, 10)+\left[c_{R}(d) \cdot \ln \left(1+\frac{a_{R}(d)}{c_{R}(d)} \cdot \frac{T-10}{10}\right)\right] \cdot Q 10_{\text {site cible }}
$$

avec $V_{R}(d, 10)$ déduit de $(4), \quad a_{R}(d)=\frac{0,209}{1+\frac{d}{\Delta_{\text {site cible }}}}$ et $c_{R}(d)=\frac{1}{4,29 \frac{d}{\Delta_{\text {site cible }}}+2,71}$.

Lorsque les gradex de pluie locaux sont disponibles, un «forçage » de la fonction régionale du gradex des pluies peut être opéré en faisant varier le paramètre $P_{2}^{*}$ pour satisfaire éventuellement aux gradex locaux, en particulier celui bien connu des pluies maximales de $24 \mathrm{~h}$. Cet aspect opérationnel est traité automatiquement dans l'application SIG [Vanderbecq, 2003]. Nous présentons à titre d'exemple (fig. 6) la fonction régionale du gradex «corrigée » pour les sous bassins de la Moselotte à Vagney (A415201) et du ruisseau du Trey à Vandières (A721301), dont quelques caractéristiques sont précisées au tableau 1 .
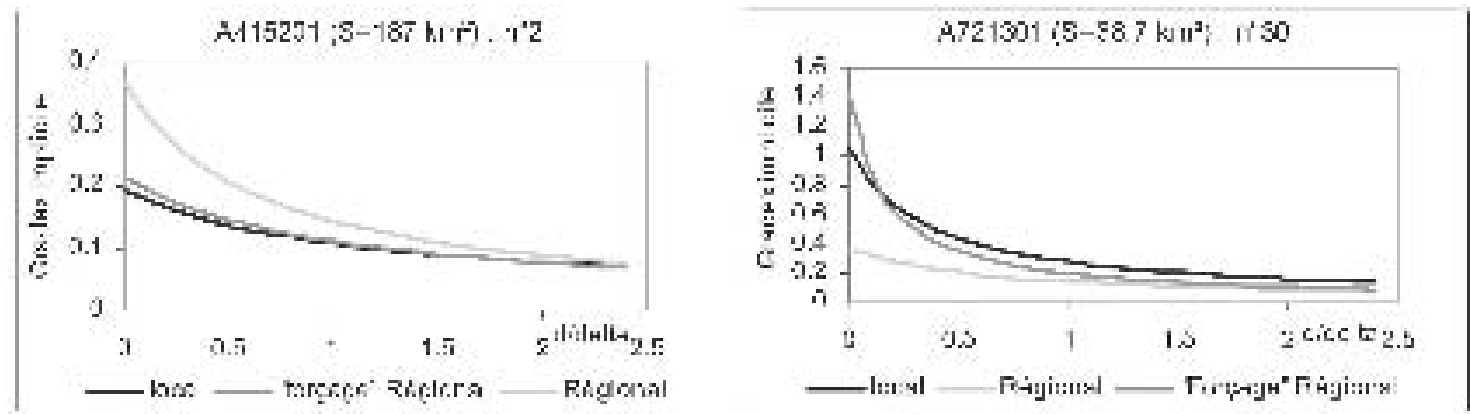

Figure 6 : « Forçage » de la fonction régionale du gradex des pluies.

Tableau 1 : Caractéristiques des sous-bassins.

\begin{tabular}{|c|c|c|c|c|c|}
\hline Code sous-bassin & Situation (figure 1) & Jeu d'appartenance & Superficie $\left(\mathrm{km}^{2}\right)$ & Delta $(\mathrm{j})$ & $Q 10\left(\mathrm{~m}^{3} / \mathrm{s}\right)$ \\
\hline A415201 & $\mathrm{n}^{\circ} 2$ & calage (27 s.b.) & 187 & 5,96 & 133 \\
\hline A721301 & $\mathrm{n}^{\circ} 30$ & validation (12 s.b.) & 38,7 & 2,54 & 6,39 \\
\hline
\end{tabular}


Les fonctions implicites du gradex des pluies ci dessus se déduisent du tableau 2.

Tableau 2 : Paramètres des fonctions implicites des gradex de pluie.

\begin{tabular}{|c|c|c|c|c|c|c|}
\hline \multirow{2}{*}{ Code } & \multirow{G}{*}{$\begin{array}{c}G p_{d=24 \mathrm{~h}}(\mathrm{~mm}) \\
\text { observé }\end{array}$} & \multicolumn{2}{|c|}{$P_{1}^{*}$} & \multicolumn{3}{c|}{$P_{2}^{*}$} \\
\cline { 3 - 7 } & & calage local & régional & calage local & régional & «forçage » \\
\hline A415201 & 11,4 & 5,11 & 4,29 & 5,56 & 2,71 & 4,7 \\
\hline A721301 & 5,90 & 2,61 & 4,29 & 0,95 & 2,71 & 0,7 \\
\hline
\end{tabular}

A ces fonctions implicites du gradex des pluies maximales, nous pouvons associer les distributions des quantiles de crue de grande période de retour respectivement modélisées (fig. 7).

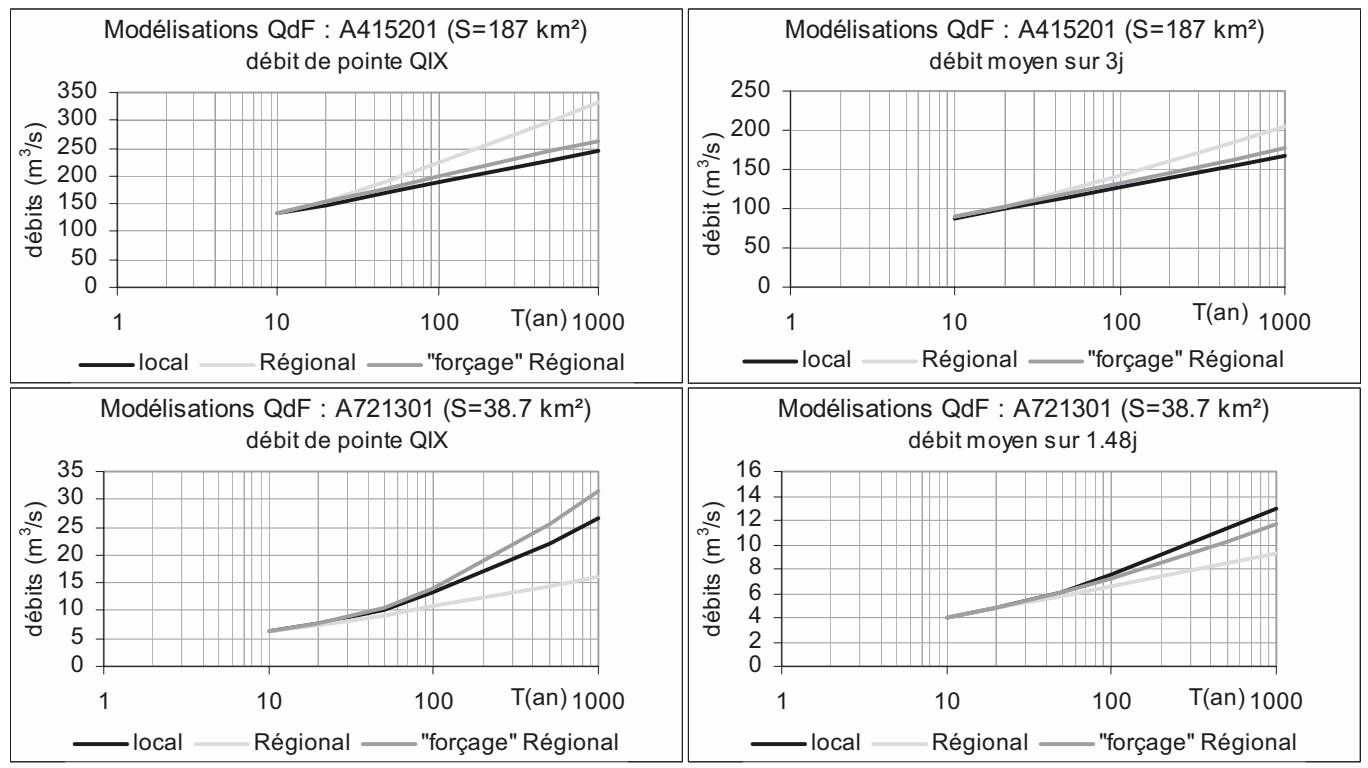

Figure 7 : Quantiles de crue associés aux fonctions implicites du gradex des pluies.

\section{ROBUSTESSE DES MODÈLES QDF RÉGIONAUX}

Les expressions (4) et (10) des deux modèles QdF régionaux permettent d'estimer respectivement les quantiles de crues fréquents et rares d'un site cible, connaissant ses deux descripteurs de régime $Q 10_{\text {site cible }}$ et $\Delta_{\text {site cible }}$. La robustesse des deux modèles régionaux est testée en comparant leurs résultats à ceux des modélisations locales respectives (2) et (7), considérées comme référence «observation». Pour que la comparaison soit objective, aucun biais n'est rentré au niveau des descripteurs de régime $Q 10_{\text {site cible }}$ et $\Delta_{\text {site cible }}$ supposés connus grâce aux observations de débit . Cette comparaison est faite pour chaque jeu de $n$ sous-bassins et un quantile de crue donné relatif à 5 durées $d(0 \leq d(\mathrm{j}) \leq 1,3 \Delta)$. Une représentation classique de cette comparaison est donnée par rapport à la première bissectrice mais aussi à partir du calcul d'une erreur quadratique moyenne (11) qui globalise l'incertitude par jeu de sous-bassins.

$$
E r_{\text {jeu }}=100 \cdot \sqrt{\frac{1}{5 n} \sum_{k=1}^{n} \sum_{i=1}^{5}\left(\frac{V_{k_{L}}\left(d_{i}, T\right)-V_{k_{R}}\left(d_{i}, T\right)}{V_{k_{L}}\left(d_{i}, T\right)}\right)^{2}}
$$

\section{VI.1 Faibles périodes de retour}

Le modèle régional (4) présente une bonne restitution des quantiles biennaux des sous bassins des trois jeux considérés (fig. 8). Nous rappelons que les écoulements des sous-bassins des jeux de calage et de validation sont indépendants et non influencés. Ceci n'est pas toujours le cas pour les sous-bassins du jeu de validation complémentaire.

\section{VI.2 Grandes périodes de retour}

La vérification porte exclusivement sur les jeux de calage et de validation, les gradex de pluies maximales de bassin n'étant pas disponibles pour le jeu de validation complémentaire. Les quantiles centennaux et millénaux sont bien restitués par le modèle QdF régional (fig. 9). 



Figure 8 : Comparaison des quantiles biennaux modélisés.
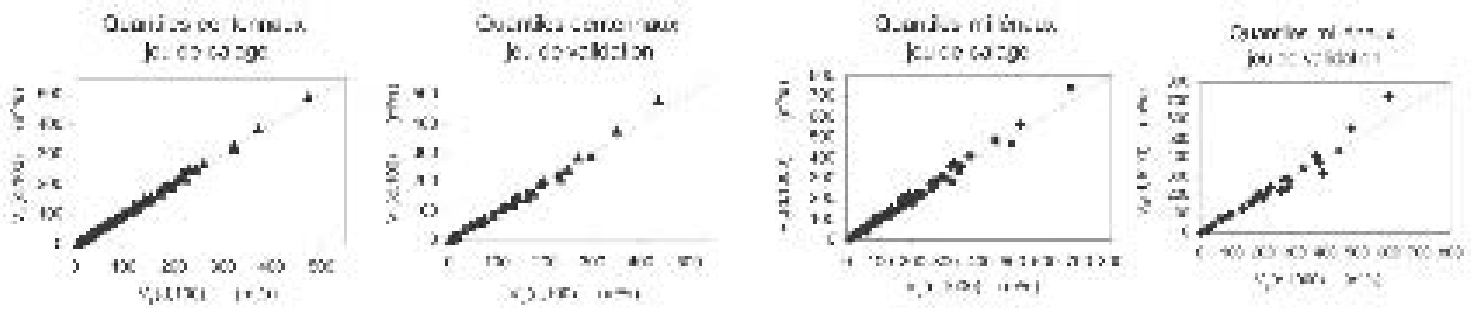

Figure 9 : Comparaison des quantiles de fréquences rares modélisés.

\section{VI.3 Graphe de synthèse}

Le graphe de synthèse de la figure 10 permet de globaliser l'incertitude (11) des modèles régionaux (4) et (10) sur l'estimation des quantiles de crues présentés précédemment (fig. 8 et 9).
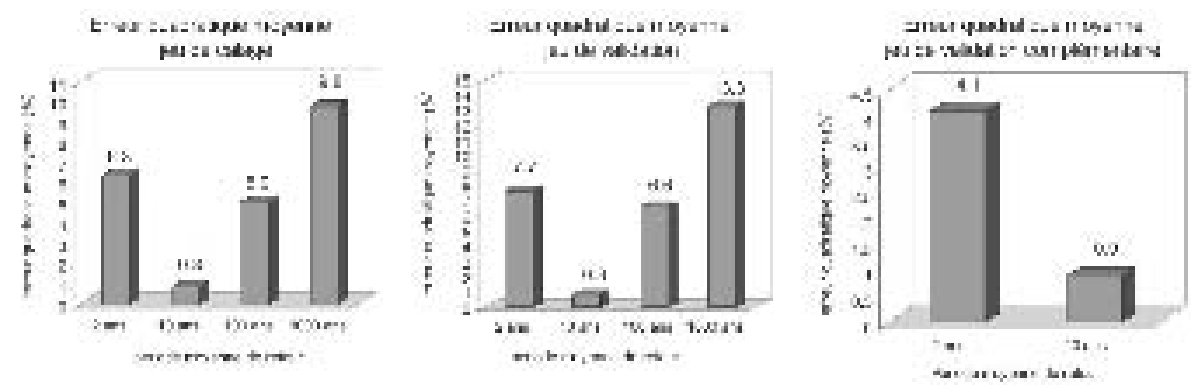

Figure 10 : Erreurs quadratiques relatives aux quantiles régionaux.

\section{VII $\square$ RÉGIONALISATION DES DESCRIPTEURS DE RÉGIME $Q 10$ ET $\Delta$ ET INCERTITUDE INDUITE SUR LES QUANTILES ESTIMÉS (4)}

\section{VII.1 Démarche «classique » : jeu de calage et jeu de validation a priori}

Les différents résultats présentés antérieurement ont permis de montrer la représentativité des modèles QdF régionaux (4) et (10) en considérant les entrées $Q 10_{\text {site cible }}$ et $\Delta_{\text {site cible }}$ connues, grâce aux observations de débit. En l'absence d'observation de débit des liens ont été établis entre ces descripteurs et certaines variables physiographiques et pluviométriques. Nous ne ferons référence qu'aux dernières relations établies pour $Q 10(12)$ et $\Delta(13)$, suite aux travaux de Galéa et al. [2001] et Blum [2003].

$$
Q 10=0,09 S^{0,84} \cdot I_{F}^{0,57} \cdot I_{b} 10 \_10^{0,91}
$$

avec $Q 10$ le débit de pointe décennal $\left(\mathrm{m}^{3} / \mathrm{s}\right), S\left(\mathrm{~km}^{2}\right), I_{F}$ l'indice de forme du sous-bassin et $I_{b} 10 \_10$ un indice d'intensité de pluie de bassin $(\mathrm{mm} / \mathrm{j})$ de durée 10 jours et décennal. 


$$
\Delta=293 \cdot S^{0,38} \cdot I_{F}^{-0,60} \cdot T O P O \_50^{-3,56}
$$

où TOPO_50 est la valeur médiane de la distribution de l'indice topographique défini par Beven et Kirkby [1979], Charleux-Demargne [2001].
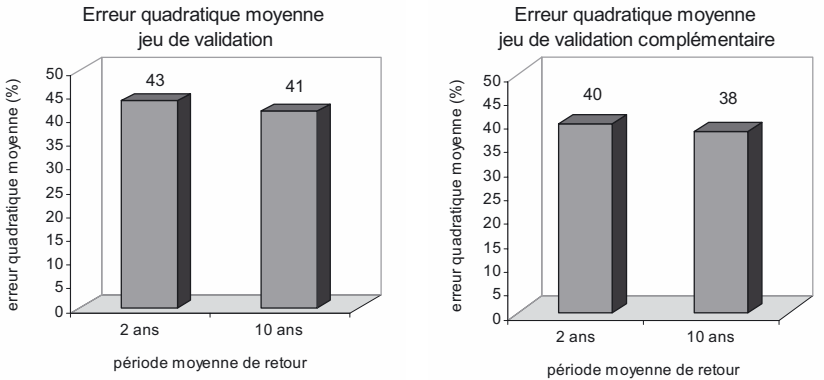

Figure 11 : Incertitude sur les quantiles régionaux (4) pour $Q 10(12)$ et $\Delta(13)$ estimés.
Le test d'erreur (11) montre que l'erreur quadratique moyenne est faible, lorsque les descripteurs de régime sont déduits des observations de débit (fig. 10). Par contre, l'incertitude encore forte sur les descripteurs estimés par régressions (12) et (13) affecte significativement les résultats du modèle régional (fig. 11).

\section{VII.2 Approche canonique du voisinage hydrologique homogène d'un site cible}

L'analyse de corrélation canonique est une technique statistique qui sert à définir les relations linéaires systématiques qui existent entre deux groupes de variables. La méthode est décrite par Saporta [1990]. Il est possible d'inférer sur les variables canoniques d'un ensemble si on connaît les variables canoniques de l'autre ensemble. Dans notre cas nous recherchons les relations de prédiction entre les variables physiographiques et les variables hydrologiques caractérisant le régime des crues des sous bassins de la Moselle (fig. 12).

$V$ sont les variables canoniques correspondantes aux variables physiographiques indépendantes $\mathrm{X}(\mathrm{X} 1, \mathrm{X} 2, \ldots, \mathrm{Xn}) . W$ sont les variables canoniques correspondantes aux variables hydrologiques dépendantes $\mathrm{Y}(\mathrm{Y} 1, \mathrm{Y} 2, \ldots, \mathrm{Yr})$.

L'application de l'analyse de corrélation canonique dans le domaine de l'hydrologie est due à Cavadias [1990]. Pour notre démarche de régionalisation des descripteurs de régime $Q 10$ et $\Delta$ [Blum, 2003], nous avons choisi l'approche proposée par Ribeiro-Corréa et al. [1995] et améliorée plus récemment par Ouarda et al. [2001]. Pour chaque sous-bassin, cette démarche comprend deux étapes : la détermination d'un voisinage hydrologique homogène par rapport au site cible et, à partir de ce voisinage hydrologique homogène, l'estimation par régression multiple des descripteurs de régime du site cible. Le voisinage (ou la région) hydrologique homogène est

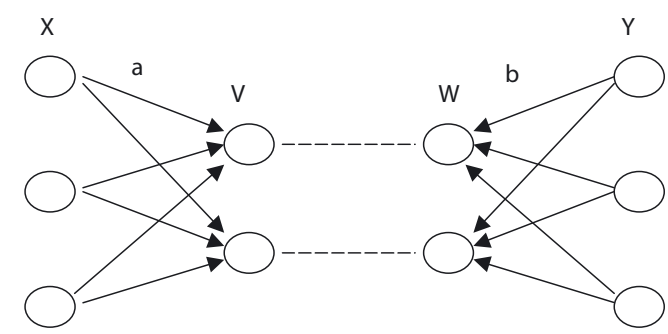

Figure 12 : Schéma de l'analyse de corrélation canonique. le regroupement de plusieurs sites ayant un certain degré de similarité avec le site cible. Ces auteurs ont proposé un algorithme pertinent pour l'identification de la région hydrologique homogène d'un site cible quelconque. Soit $W$ les variables canoniques correspondantes aux variables hydrologiques dépendantes $Y, V$ : les variables canoniques correspondantes aux variables physiographiques indépendantes $X$ et $\lambda_{i}, i=1, \ldots, p$ les $p$ corrélations canoniques obtenues à la suite de l'analyse de corrélation canonique. Soit $I_{p}$ la matrice d'identité $p \times p$ et $\Lambda$ la matrice diagonale qui est formée avec les valeurs des $p$ corrélations canoniques. En supposant la normalité du vecteur $\left(\begin{array}{l}W \\ V\end{array}\right)$, la distribution conditionnelle de

$W$ étant donné $V$ est aussi normale. La conséquence est que les sous bassins similaires du point de vue physiographique seront éparpillés dans l'espace canonique des variables hydrologiques autour d'une position moyenne $\Lambda V$. Ainsi, selon Ribeiro-Corréa [1995], la constitution de la région hydrologique homogène repose sur le calcul d'une distance entre les deux variables canoniques $V$ et $W$. Soit $v_{0}$ le score canonique physiographique qui correspond au sous-bassin cible et $w$ une réalisation de $W$ (correspondant à un autre sous-bassin). La distance de Mahalanobis $D^{2}=\left(w-\Lambda v_{0}\right)^{\prime}\left(I_{p}-\Lambda^{2}\right)^{-1}\left(w-\Lambda v_{0}\right)$ nous donne une mesure de la similarité entre les deux sous-bassins. Blum [2003] a considéré 50 sous-bassins au lieu de 53 (information non disponible). L'algorithme de Ribeiro-Corréa a été modifié pour prendre en compte des voisinages de taille fixe. Ainsi, pour chaque sous-bassin, nous avons calculé les réalisations $\left(w_{i}-v_{0}\right)^{\prime}\left(I_{p}-\Lambda^{2}\right)^{-1}\left(w_{i}-v_{0}\right), i=1, \ldots, 49$ correspondant aux distances entre le sous-bassin cible et chacun des sous-bassins restants. Les sites qui correspondent aux plus petites des $K$ valeurs forment le voisinage de taille $K$ du sous-bassin cible. Ceci nous a permis d'évaluer objectivement l'impact de la taille des voisinages sur la qualité des estimations par régression multiple. Nous avons fait varier cette taille de 7 sites voisinants (taille minimale) à 49 (tous les sous-bassins 
sont inclus dans le voisinage). Le test d'erreur (14) varie en fonction de la taille $K$ de la région hydrologique homogène (fig. 14).

$$
r E Q(\text { taille }) \%=100 \cdot \sqrt{\frac{1}{K} \sum_{i=1}^{i=k}\left[\frac{\left(\hat{x}_{i, \text { taille }}-x_{i}\right)}{x_{i}}\right]^{2}}
$$

où $r E Q$ (taille) est l'erreur quadratique moyenne $(\%), K$ le nombre total de sous bassins, $x_{i}$ la valeur observée du descripteur de régime au site $i$ et $\hat{x}_{i \text {,taille }}$ la valeur estimée du descripteur de régime au site $i$, pour un voisinage hydrologique homogène dont le nombre de stations voisinantes permet de définir sa taille. Pour $\Delta$ et $Q 10$ sont conservées les trois mêmes variables explicatives : $S, T O P O$ et $I_{b} 10 \_10$. Pour $\Delta$, la taille optimale de la zone hydrologique homogène est de 19 sous-bassins. Le modèle linéaire d'estimation de $\Delta$ pour un site cible donné est calé à partir des 19 stations voisinantes qui constituent la région hydrologique homogène identifiée par l'analyse de corrélation canonique.

Pour $Q 10$, la taille de la région hydrologique définie comme homogène concerne l'ensemble des sous-bassins retenus, en fait 47 si l'on enlève les trois plus influents selon la distance de Cook [Rousseuw et Leroy, 1987].

Nous avons comparé les résultats de l'approche canonique aux résultats présentés antérieurement. Un seul jeu de validation de 24 sous-bassins a été considéré. Pour $Q 10$ et $\Delta$, les régions hydrologiques homogènes identifiées pour les 24 sous-bassins permettent une nette amélioration par rapport aux estimations respectives (12) et (13) sans voisinage défini (fig. 15).

L'approche par voisinage homogène a permis une amélioration de l'ordre de $50 \%$ de l'erreur quadratique moyenne sur l'estimation de $Q 10$ et de $33 \%$ pour l'estimation de $\Delta$ (tab. 3).
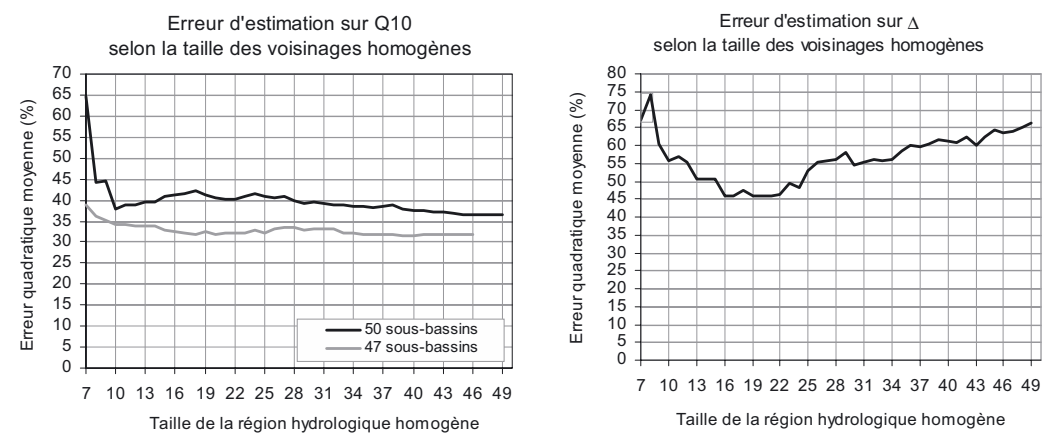

Figure 14 : Définition de la taille optimale de la région hydrologique homogène pour $Q 10$ et $\Delta$.
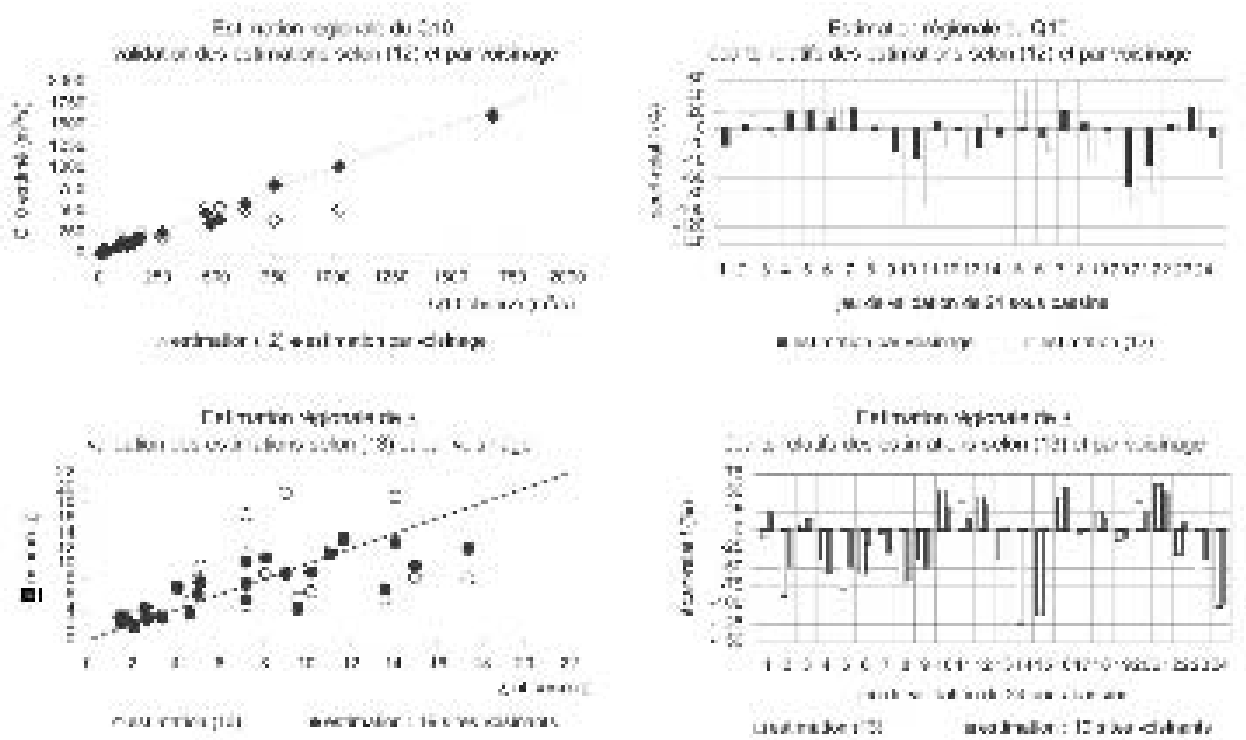

Figure 15 : Estimation régionale des descripteurs et écart relatif par rapport à l'observation.

Tableau 3 : Incertitude d'estimation des descripteurs de régime.

\begin{tabular}{|c|c|c|c|c|c|}
\hline Modèle linéaire d'estimation & \multicolumn{2}{|c|}{ selon (12) et (13) } & \multicolumn{2}{c|}{ a. canonique avec 50 s.b. } & a. canonique avec 47 s.b. \\
\hline Descripteurs de régime & $Q 10(12)$ & $\Delta(13)$ & $Q 10$ & $\Delta$ & $Q 10$ \\
\hline erreur quadratique moyenne (\%) & 47 & 60 & 25 & 40 & 23 \\
\hline
\end{tabular}



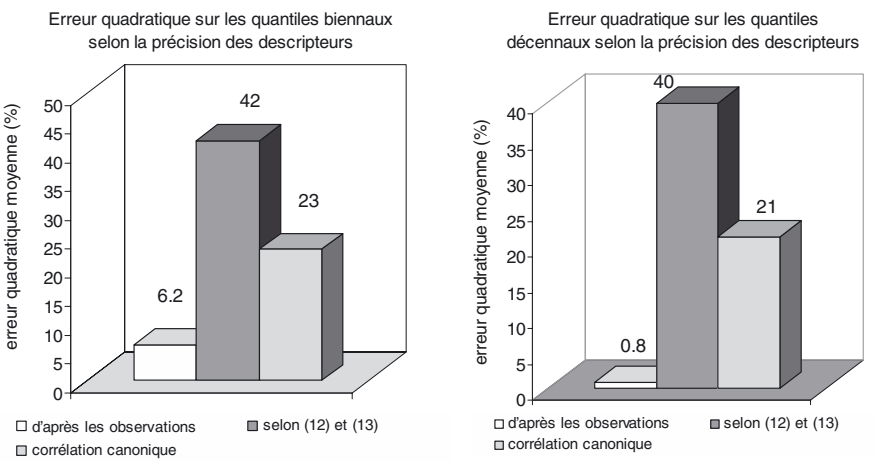

Figure 16 : Incertitude sur les quantiles de crue régionaux (4) selon la précision des descripteurs.

Cette amélioration de l'estimation des descripteurs de régime se traduit par un gain d'environ $50 \%$ de l'erreur quadratique (11) sur les quantiles de crue du jeu de validation de 24 sous-bassins (fig. 16).

\section{VIII $\square$ CONCLUSION}

Les modèles QdF régionaux (4) et (10) permettent de caractériser le régime des crues d'un site cible quelconque du bassin hydrographique de la Moselle française. Les quantiles de crues modélisés sont d'autant plus probants que les descripteurs de régime $Q 10_{\text {site cible }}$ et $\Delta_{\text {site cible }}$ sont observés ou estimés avec suffisamment de précision. Une nette amélioration des modèles linéaires d'estimation a pu être obtenue grâce à l'analyse de corrélation canonique qui permet d'identifier pour chaque site cible les sous bassins appartenant à la région hydrologique homogène. Pour le jeu de validation de 24 sous bassins considéré, l'amélioration de l'erreur quadratique moyenne est d'environ $50 \%$ pour $Q 10$ et $33 \%$ pour $\Delta$. Cela induit sur les quantiles de crue modélisés (4), pour différentes durées, une amélioration de $50 \%$ environ de l'erreur quadratique moyenne. L'analyse de corrélation canonique permet donc de satisfaire à une objectivité relative des modélisations $\mathrm{QdF}$ régionales proposées.

\section{IX — RÉFÉRENCES ET CITATIONS}

Beven, K.J., \& KiRKBy, M.J. 1979. A physically based variable contributing area model of basin hydrology. Hydrological Sciences Bulletin, 24(1), 43-69.

BLuM, M. 2003. La détermination de régions hydrologiques homogènes par l'analyse de corrélation canonique. DESS SITN, soutenu le 17 septembre 2003 à l'Université Claude Bernard, Lyon 1. UR Hydrologie-Hydraulique, Cemagref Groupement de Lyon.

CAVAdias, G.S. 1990. The canonical correlation approach to regional flood estimation. Regionalization in Hydrology, Proc. Of the Ljubljana Symposium, IAHS, Publ., 191, 171-177.

CharleuX-Demargne, J. 2001. Qualité des modèles numériques de terrain pour l'hydrologie. Application à la caractérisation du régime de crues des bassins versants. UMR $3 \mathrm{~S}$ «Structures et systèmes spatiaux » Cemagref-Engref, Montpellier. Thèse soutenue le 29 juin 2001 (Université de Marne-la-Vallée).

Dalrymple, T. 1960. Flood frequency analysis. U.S. Geol. Surv. Water Supply Pap., 1543A.
Galéa, G., Gendreau, N., Javelle, P., Poinard, D., Puech, C., \& Schneider, M. 2001. Régimes des crues des sous bassins de la Moselle française / modèles régionaux et SIG associé. Projet PACTES, UR Hydrologie-Hydraulique, Cemagref-Lyon.

Guillot, P., \& DubAND, D. 1967. La méthode du GRADEX pour le calcul de la probabilité des crues à partir des pluies. Journées de la SHF (septembre), question 1, rapport 7, Paris.

Javelle, P., Grésillon, J.M., \& GaléA, G. 1999. Modélisation des courbes Débit-durée-Fréquence en crues et invariance d'échelle. Comptes Rendus de l'Académie des Sciences Paris, Sciences de la terre et des planètes / 329, 39-44.

Javelle, P., GalÉa, G., \& Grésillon, J.M. 2000. L’approche débit-durée-fréquence, historique et avancées, Revue des Sciences de l'Eau, 13(3), 305-323.

JAvelLe, P. 2001. Caractérisation du régime des crues : le modèle débit-durée-fréquence convergent - Approche locale et régionale. UR Hydrologie-Hydraulique, Cemagref, groupement de Lyon, thèse soutenue le 01.03.2001 à l' INPG (MMGE).

LAnG, M., OuARdA, T.B.M.J., \& BobÉE, B. 1999. Towards operational guidelines for over-threshold modeling. Journal of Hydrology, 225, 103-117.

Michel, C., \& Oberlin, G. 1987. Seuil d'application de la méthode du GRADEX. La Houille Blanche n 3-1987, 199-203.

NOAH, 2000. New Opportunities for Altimetry in Hydrology Final Report, European Commission DG XII, Environment and Climat, Theme 3, Space and Techniques, CEO, ref. NOAH ENV4-CT96-0371, 318 p.

Ouarda, T., Girard, C., Cavadias, G.S., \& BobéE, B. 2001. Regional flood frequency estimation with canonical correlation analysis, Journal of Hydrology, 254(1-4): 157-173.

PACTES, 2001. Séminaire prévention au Cemagref de Montpellier, 5-6 juin 2001. Cemagref, groupement de Lyon, UR Hydrologie-Hydraulique.

Ribeiro-Corréa, J., Cavadias, G.S., Clément, B., \& Rousselle, J. 1995. Identification of hydrological neighborhoods using canonical correlation analysis, Journal of Hydrology, 173: 71-89.

Rousseuw, J.P., \& Leroy, A.M. 1987. Robust regression and outlier detection, John Wiley \& Sons.

SAPORTA, G. 1990. Probabilité, analyse de données et statistique, Editions technip.

VANDERBECQ, A. 2003. Réalisation d'une application métier S.I.G. Intégration du modèle QdF sous Arc View 3.2. DESS : Application de l'informatique aux Sciences de la Terre, soutenu le 23.10.2003 à l'ENSG, Marne-la-Vallée. 